\title{
Erratum: "Searches for Gravitational Waves from Known Pulsars at Two Harmonics in 2015-2017 LIGO Data" (2019, ApJ, 879, 10)
}

B. P. Abbott ${ }^{1}$, R. Abbott ${ }^{1}$, T. D. Abbott ${ }^{2}$, S. Abraham ${ }^{3}$, F. Acernese ${ }^{4,5}$, K. Ackley ${ }^{6}$, C. Adams $^{7}$, R. X. Adhikari ${ }^{1}$, V. B. Adya ${ }^{8,9}$, C. Affeldt ${ }^{8,9}$, M. Agathos ${ }^{10}$, K. Agatsuma ${ }^{11}$, N. Aggarwal ${ }^{12}$, O. D. Aguiar ${ }^{13}$, L. Aiello ${ }^{14,15}$, A. Ain ${ }^{3}$, P. Ajith ${ }^{16}$, G. Allen ${ }^{17}$, A. Allocca ${ }^{18,19}$, M. A. Aloy ${ }^{20}$, P. A. Altin ${ }^{21}$, A. Amato ${ }^{22}$, A. Ananyeva ${ }^{1}$, S. B. Anderson ${ }^{1}$, W. G. Anderson ${ }^{23}$, S. V. Angelova ${ }^{24}$, S. Antier ${ }^{25}$, S. Appert ${ }^{1}$, K. Arai ${ }^{1}$, M. C. Araya ${ }^{1}$, J. S. Areeda ${ }^{26}$, M. Arène ${ }^{27}$, N. Arnaud ${ }^{25,28}$, S. Ascenzi ${ }^{29,30}$, G. Ashton ${ }^{6}$, S. M. Aston ${ }^{7}$, P. Astone ${ }^{31}$, F. Aubin ${ }^{32}$, P. Aufmuth ${ }^{9}$, K. AultONeal ${ }^{33}$, C. Austin ${ }^{2}$, V. Avendano ${ }^{34}$, A. Avila-Alvarez ${ }^{26}$,

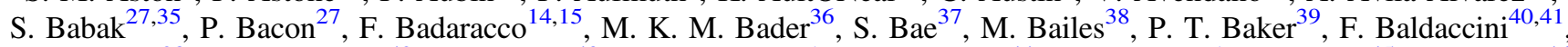
G. Ballardin ${ }^{28}$, S. W. Ballmer ${ }^{42}$, S. Banagiri ${ }^{43}$, J. C. Barayoga ${ }^{1}$, S. E. Barclay ${ }^{44}$, B. C. Barish ${ }^{1}$, D. Barker ${ }^{45}$, K. Barkett $^{46}$, S. Barnum ${ }^{12}$, F. Barone ${ }^{4,5}$, B. Barr ${ }^{44}$, L. Barsotti ${ }^{12}$, M. Barsuglia ${ }^{27}$, D. Barta ${ }^{47}$, J. Bartlett ${ }^{45}$, I. Bartos ${ }^{48}$, R. Bassiri ${ }^{49}$, A. Basti ${ }^{18,19}$, M. Bawaj ${ }^{41,50}$, J. C. Bayley ${ }^{44}$, M. Bazzan ${ }^{51,52}$, B. Bécsy ${ }^{53}$, M. Bejger ${ }^{27,54}$, I. Belahcene ${ }^{25}$, A. S. Bell ${ }^{44}$, D. Beniwal ${ }^{55}$, B. K. Berger ${ }^{49}$, G. Bergmann ${ }^{8,9}$, S. Bernuzzi ${ }^{56,57}$, J. J. Bero ${ }^{58}$, C. P. L. Berry ${ }^{59}$, D. Bersanetti ${ }^{60}$, A. Bertolini ${ }^{36}$, J. Betzwieser ${ }^{7}$, R. Bhandare ${ }^{61}$, J. Bidler ${ }^{26}$, I. A. Bilenko ${ }^{62}$, S. A. Bilgili ${ }^{39}$, G. Billingsley ${ }^{1}$, J. Birch ${ }^{7}$, R. Birney ${ }^{24}$, O. Birnholtz ${ }^{58}$, S. Biscans ${ }^{1,12}$, S. Biscoveanu ${ }^{6}$, A. Bisht ${ }^{9}$, M. Bitossi ${ }^{19,28}$, M. A. Bizouard ${ }^{25}$, J. K. Blackburn ${ }^{1}$, C. D. Blair ${ }^{7}$, D. G. Blair ${ }^{63}$, R. M. Blair ${ }^{45}$, S. Bloemen ${ }^{64}$, N. Bode ${ }^{8,9}$, M. Boer ${ }^{65}$, Y. Boetzel ${ }^{66}$, G. Bogaert ${ }^{65}$, F. Bondu ${ }^{67}$, E. Bonilla ${ }^{49}$, R. Bonnand ${ }^{32}$, P. Booker ${ }^{8,9}$, B. A. Boom ${ }^{36}$, C. D. Booth ${ }^{68}$, R. Bork ${ }^{1}$, V. Boschi ${ }^{28}$, S. Bose ${ }^{3,69}$, K. Bossie ${ }^{7}$, V. Bossilkov ${ }^{63}$, J. Bosveld ${ }^{63}$, Y. Bouffanais ${ }^{27}$, A. Bozzi ${ }^{28}$, C. Bradaschia ${ }^{19}$, P. R. Brady ${ }^{23}$, A. Bramley ${ }^{7}$, M. Branchesi ${ }^{14,15}$, J. E. Brau ${ }^{70}$, T. Briant ${ }^{71}$, J. H. Briggs ${ }^{44}$, F. Brighenti ${ }^{72,73}$, A. Brillet ${ }^{65}$, M. Brinkmann ${ }^{8,9}$, V. Brisson ${ }^{25,191}$, P. Brockill ${ }^{23}$, A. F. Brooks ${ }^{1}$, D. D. Brown ${ }^{55}$, S. Brunett ${ }^{1}$, A. Buikema ${ }^{12}$, T. Bulik ${ }^{74}$, H. J. Bulten ${ }^{36,75}$, A. Buonanno ${ }^{35,76}$, D. Buskulic ${ }^{32}$, C. Buy ${ }^{27}$, R. L. Byer ${ }^{49}$, M. Cabero ${ }^{8,9}$, L. Cadonati ${ }^{77}$, G. Cagnoli ${ }^{22,78}$, C. Cahillane ${ }^{1}$, J. Calderón Bustillo ${ }^{6}$, T. A. Callister ${ }^{1}$, E. Calloni ${ }^{5,79}$, J. B. Camp ${ }^{80}$, W. A. Campbell ${ }^{6}$,

M. Canepa ${ }^{60,81}$, K. C. Cannon ${ }^{82}$, H. Cao ${ }^{55}$, J. Cao ${ }^{83}$, E. Capocasa ${ }^{27}$, F. Carbognani ${ }^{28}$, S. Caride ${ }^{84}$, M. F. Carney ${ }^{59}$, G. Carullo ${ }^{18}$, J. Casanueva Diaz ${ }^{19}$, C. Casentini2 ${ }^{29,30}$, S. Caudill ${ }^{36}$, M. Cavaglià ${ }^{85}$, F. Cavalier ${ }^{25}$, R. Cavalieri ${ }^{28}$, G. Cella ${ }^{19}$, P. Cerdá-Durán ${ }^{20}$, G. Cerretani $^{18,19}$, E. Cesarini ${ }^{30,86}$, O. Chaibi ${ }^{65}$, K. Chakravarti ${ }^{3}$, S. J. Chamberlin ${ }^{87}$, M. Chan ${ }^{44}$, S. Chao ${ }^{88}$, P. Charlton ${ }^{89}$,

E. A. Chase ${ }^{59}$, E. Chassande-Mottin ${ }^{27}$, D. Chatterjee ${ }^{23}$, M. Chaturvedi ${ }^{61}$, B. D. Cheeseboro ${ }^{39}$, H. Y. Chen ${ }^{90}$, X. Chen ${ }^{63}$, Y. Chen $^{46}$, H.-P. Cheng ${ }^{48}$, C. K. Cheong ${ }^{91}$, H. Y. Chia ${ }^{48}$, A. Chincarini ${ }^{60}$, A. Chiummo ${ }^{28}$, G. Cho ${ }^{92}$, H. S. Cho ${ }^{93}$, M. Cho $^{76}$,

N. Christensen ${ }^{65,94}$, Q. Chu ${ }^{63}$, S. Chua ${ }^{71}$, K. W. Chung ${ }^{91}$, S. Chung ${ }^{63}$, G. Ciani ${ }^{51,52}$, A. A. Ciobanu ${ }^{55}$, R. Ciolfi ${ }^{95,96}$, F. Cipriano ${ }^{65}$, A. Cirone ${ }^{60,81}$, F. Clara ${ }^{45}$, J. A. Clark ${ }^{77}$, P. Clearwater ${ }^{97}$, F. Cleva ${ }^{65}$, C. Cocchieri ${ }^{85}$, E. Coccia ${ }^{14,15}$, P.-F. Cohadon ${ }^{71}$, D. Cohen ${ }^{25}$, R. Colgan ${ }^{98}$, M. Colleoni ${ }^{99}$, C. G. Collette ${ }^{100}$, C. Collins ${ }^{11}$, L. R. Cominsky ${ }^{101}$, M. Constancio, Jr. ${ }^{13}$, L. Conti ${ }^{52}$, S. J. Cooper ${ }^{11}$, P. Corban ${ }^{7}$, T. R. Corbitt ${ }^{2}$, I. Cordero-Carrión ${ }^{102}$, K. R. Corley ${ }^{98}$, N. Cornish ${ }^{53}$, A. Corsi ${ }^{84}$, S. Cortese $^{28}$, C. A. Costa ${ }^{13}$, R. Cotesta $^{35}$,

M. W. Coughlin ${ }^{1}$, S. B. Coughlin ${ }^{59,68}$, J.-P. Coulon ${ }^{65}$, S. T. Countryman ${ }^{98}$, P. Couvares ${ }^{1}$, P. B. Covas ${ }^{99}$, E. E. Cowan ${ }^{77}$,

D. M. Coward ${ }^{63}$, M. J. Cowart ${ }^{7}$, D. C. Coyne ${ }^{1}$, R. Coyne ${ }^{103}$, J. D. E. Creighton ${ }^{23}$, T. D. Creighton ${ }^{104}$, J. Cripe ${ }^{2}$, M. Croquette ${ }^{71}$, S. G. Crowder ${ }^{105}$, T. J. Cullen ${ }^{2}$, A. Cumming ${ }^{44}$, L. Cunningham ${ }^{44}$, E. Cuoco ${ }^{28}$, T. Dal Canton ${ }^{80}$, G. Dálya ${ }^{106}$, S. L. Danilishin ${ }^{8,9}$, S. D’Antonio ${ }^{30}$, K. Danzmann ${ }^{8,9}$, A. Dasgupta ${ }^{107}$, C. F. Da Silva Costa ${ }^{48}$, L. E. H. Datrier ${ }^{44}$, V. Dattilo ${ }^{28}$, I. Dave ${ }^{61}$, M. Davier ${ }^{25}$, D. Davis ${ }^{42}$, E. J. Daw ${ }^{108}$, D. DeBra ${ }^{49}$, M. Deenadayalan ${ }^{3}$, J. Degallaix ${ }^{22}$, M. De Laurentis ${ }^{5,79}$, S. Deléglise ${ }^{71}$, W. Del Pozzo ${ }^{18,19^{\prime}}$, L. M. DeMarchi ${ }^{59}$, N. Demos ${ }^{12}$, T. Dent ${ }^{8,9,109}$, R. De Pietri ${ }^{57,10}$, J. Derby ${ }^{26}$, R. De Rosa ${ }^{5,79}$, C. De Rossi ${ }^{22,28}$, R. DeSalvo ${ }^{111}$, O. de Varona ${ }^{8,9}$, S. Dhurandhar ${ }^{3}$, M. C. Díaz ${ }^{104}$, T. Dietrich ${ }^{36}$, L. Di Fiore ${ }^{5}$, M. Di Giovanni ${ }^{96,112}$, T. Di Girolamo ${ }^{5,79}$, A. Di Lieto ${ }^{18,19}$, B. Ding ${ }^{100}$, S. Di Pace ${ }^{31,113}$, I. Di Palma ${ }^{31,113}$, F. Di Renzo ${ }^{18,19}$, A. Dmitriev ${ }^{11}$, Z. Doctor ${ }^{90}$, F. Donovan ${ }^{12}$, K. L. Dooley ${ }^{68,85}$, S. Doravari ${ }^{8,9}$, I. Dorrington ${ }^{68}$, T. P. Downes ${ }^{23}$, M. Drago ${ }^{14,15}$, J. C. Driggers ${ }^{45}$, Z. Du ${ }^{83}$, J.-G. Ducoin ${ }^{25}$, P. Dupej ${ }^{44}$, S. E. Dwyer ${ }^{45}$, P. J. Easter ${ }^{6}$, T. B. Edo ${ }^{108}$, M. C. Edwards ${ }^{94}$, A. Effler ${ }^{7}$, P. Ehrens ${ }^{1}$, J. Eichholz ${ }^{1}$, S. S. Eikenberry ${ }^{48}$, M. Eisenmann ${ }^{32}$, R. A. Eisenstein ${ }^{12}$, R. C. Essick ${ }^{90}$, H. Estelles ${ }^{99}$, D. Estevez ${ }^{32}$, Z. B. Etienne ${ }^{39}$, T. Etzel ${ }^{1}$, M. Evans ${ }^{12}$,

T. M. Evans ${ }^{7}$, V. Fafone ${ }^{14,29,30}$, H. Fair ${ }^{42}$, S. Fairhurst ${ }^{68}$, X. Fan $^{83}$, S. Farinon ${ }^{60}$, B. Farr ${ }^{70}$, W. M. Farr ${ }^{11}$, E. J. Fauchon-Jones ${ }^{68}$, M. Favata ${ }^{34}$, M. Fays ${ }^{108}$, M. Fazio ${ }^{114}$, C. Fee ${ }^{115}$, J. Feicht ${ }^{1}$, M. M. Fejer ${ }^{49}$, F. Feng ${ }^{27}$, A. Fernandez-Galiana ${ }^{2}$, I. Ferrante ${ }^{18,19}$,

E. C. Ferreira ${ }^{13}$, T. A. Ferreira ${ }^{13}$, F. Ferrini ${ }^{28}$, F. Fidecaro ${ }^{18,19}$, I. Fiori ${ }^{28}$, D. Fiorucci' ${ }^{27}$, M. Fishbach ${ }^{90}$, R. P. Fisher ${ }^{42,116}$,

J. M. Fishner ${ }^{12}$, M. Fitz-Axen ${ }^{43}$, R. Flaminio ${ }^{32,117}$, M. Fletcher ${ }^{44}$, E. Flynnn ${ }^{26}$, H. Fong ${ }^{118}$, J. A. Font ${ }^{20,119}$, P. W. F. Forsyth ${ }^{21}$, J.-D. Fournier ${ }^{65}$, S. Frasca ${ }^{31,113}$, F. Frasconi ${ }^{19}{ }^{3}$, Z. Frei ${ }^{106}$, A. Freise ${ }^{11}$, R. Frey ${ }^{70}$, V. Frey ${ }^{25}$, P. Fritschel ${ }^{12}$, V. V. Frolov ${ }^{7}$, P. Fulda ${ }^{48}$, M. Fyffe ${ }^{7}$, H. A. Gabbard ${ }^{44}$, B. U. Gadre ${ }^{3}$, S. M. Gaebel' ${ }^{11}$, J. R. Gair ${ }^{120}$, L. Gammaitoni ${ }^{40}$, M. R. Ganija ${ }^{55}$, S. G. Gaonkar ${ }^{3}$, A. Garcia ${ }^{26}$, C. García-Quirós ${ }^{99}$, F. Garufi ${ }^{5,79}$, B. Gateley ${ }^{45}$, S. Gaudio ${ }^{33}$, G. Gaur ${ }^{121}$, V. Gayathri ${ }^{122}$, G. Gemme ${ }^{60}$, E. Genin $^{28}$, A. Gennai ${ }^{19}$, D. George ${ }^{17}$, J. George ${ }^{61}$, L. Gergely ${ }^{123}$, V. Germain ${ }^{32}$, S. Ghonge ${ }^{77}$, Abhirup Ghosh ${ }^{16}$, Archisman Ghosh ${ }^{36}$, S. Ghosh ${ }^{23}$, B. Giacomazzo ${ }^{96,112}$, J. A. Giaime ${ }^{2,7}$, K. D. Giardina ${ }^{7}$, A. Giazotto ${ }^{19,192}$, K. Gill ${ }^{33}$, G. Giordano ${ }^{4,5}$, L. Glover ${ }^{111}$, P. Godwin ${ }^{87}$, E. Goetz ${ }^{45}$, R. Goetz ${ }^{48}$, B. Goncharov ${ }^{6}$, G. González ${ }^{2}$, J. M. Gonzalez Castro ${ }^{18,19}$, A. Gopakumar ${ }^{124}$,

M. L. Gorodetsky ${ }^{62}$, S. E. Gossan ${ }^{1}$, M. Gosselin ${ }^{28}$, R. Gouaty ${ }^{32}$, A. Grado ${ }^{5,125}$, C. Graef ${ }^{44}$, M. Granata ${ }^{22}$, A. Grant ${ }^{44}$, S. Gras $^{12}$, P. Grassia ${ }^{1}$, C. Gray ${ }^{45}$, R. Gray ${ }^{44}$, G. Greco ${ }^{72,73}$, A. C. Green ${ }^{11,48}$, R. Green ${ }^{68}$, E. M. Gretarsson ${ }^{33}$, P. Groot ${ }^{64}$, H. Grote ${ }^{68}$, S. Grunewald ${ }^{35}$, P. Gruning ${ }^{25}$, G. M. Guidi ${ }^{72,73}$, H. K. Gulati ${ }^{107}$, Y. Guo ${ }^{36}$, A. Gupta ${ }^{87}$, M. K. Gupta ${ }^{107}$, E. K. Gustafson ${ }^{1}$, R. Gustafson ${ }^{126}$, L. Haegel ${ }^{99}$, O. Halim ${ }^{14,15}$, B. R. Hall ${ }^{69}$, E. D. Hall ${ }^{12}$, E. Z. Hamilton ${ }^{68}$, G. Hammond ${ }^{44}$, M. Haney ${ }^{66}$, 


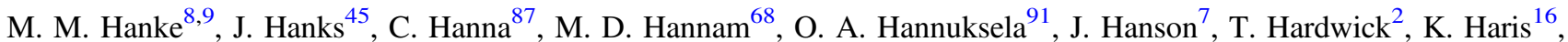
J. Harms ${ }^{14,15}$, G. M. Harry ${ }^{127}$, I. W. Harry ${ }^{35}$, C.-J. Haster ${ }^{18}$, K. Haughian ${ }^{44}$, F. J. Hayes ${ }^{44}$, J. Healy ${ }^{58}$, A. Heidmann ${ }^{71}$, M. C. Heintze ${ }^{7}$, H. Heitmann ${ }^{65}$, P. Hello ${ }^{25}$, G. Hemming ${ }^{28}$, M. Hendry ${ }^{44}$ I. S. Heng ${ }^{4}$, J. Hennig ${ }^{8,9}$, A. W. Heptonstall ${ }^{1}$, Francisco Hernandez Vivanco ${ }^{6}$, M. Heurs ${ }^{8,9}$, S. Hild ${ }^{44}$, T. Hinderer ${ }^{36,128,129}$, W. C. G. Ho ${ }^{130}$, D. Hoak ${ }^{28}$, S. Hochheim ${ }^{8,9}$, D. Hofman ${ }^{22}$, A. M. Holgado ${ }^{17}$, N. A. Holland ${ }^{21}$, K. Holt ${ }^{7}$, D. E. Holz ${ }^{90}$, P. Hopkins ${ }^{68}$, C. Horst ${ }^{23}$, J. Hough ${ }^{44}$, E. J. Howell ${ }^{63}$, C. G. Hoy ${ }^{68}$, A. Hreibi ${ }^{65}$, E. A. Huerta ${ }^{17}$, D. Huet ${ }^{25}$, B. Hughey ${ }^{33}$, M. Hulko ${ }^{1}$, S. Husa ${ }^{99}$, S. H. Huttner ${ }^{44}$, T. Huynh-Dinh ${ }^{7}$, B. Idzkowski ${ }^{74}$, A. Iess ${ }^{29,30}$, C. Ingram ${ }^{55}$, R. Inta ${ }^{84}$, G. Intini ${ }^{31113}$, B. Irwin ${ }^{115}$, H. N. Isa ${ }^{44}$, J.-M. Isac ${ }^{71}$, M. Isi ${ }^{1}$, B. R. Iyer ${ }^{16}$, K. Izumi $^{45}$, T. Jacqmin ${ }^{71}$, S. J. Jadhav ${ }^{131}$, K. Jani ${ }^{77}$, N. N. Janthalur ${ }^{131}$, P. Jaranowski ${ }^{132}$, A. C. Jenkins ${ }^{133}$, J. Jiang ${ }^{48}$,

D. S. Johnson ${ }^{17}$, A. W. Jones ${ }^{11}$, D. I. Jones ${ }^{134}$, R. Jones ${ }^{44}$, R. J. G. Jonker ${ }^{36}$, L. Ju ${ }^{63}$, J. Junker ${ }^{8,9}$, C. V. Kalaghatgi ${ }^{68}$, V. Kalogera ${ }^{59}$, B. Kamai ${ }^{1}$, S. Kandhasamy ${ }^{85}$, G. Kang ${ }^{37}$, J. B. Kanner ${ }^{1}$, S. J. Kapadia ${ }^{23}$, S. Karki ${ }^{70}$, K. S. Karvinen ${ }^{8,9}$, R. Kashyap ${ }^{16}$,

M. Kasprzack ${ }^{1}$, S. Katsanevas ${ }^{28}$, E. Katsavounidis ${ }^{12}$, W. Katzman ${ }^{7}$, S. Kaufer ${ }^{9}$, K. Kawabe ${ }^{45}$, N. V. Keerthana ${ }^{3}$, F. Kéfélian ${ }^{65}$, D. Keitel ${ }^{44}$, R. Kennedy ${ }^{108}$, J. S. Key ${ }^{135}$, F. Y. Khalili ${ }^{62}$, H. Khan ${ }^{26}$, I. Khan ${ }^{14,30}$, S. Khan ${ }^{8,9}$, Z. Khan ${ }^{107}$, E. A. Khazanov ${ }^{136}$, M. Khursheed ${ }^{61}$, N. Kijbunchoo ${ }^{21}$, Chunglee Kim ${ }^{137}$, J. C. Kim ${ }^{138}$, K. Kim ${ }^{91}$, W. Kim ${ }^{55}$, W. S. Kim ${ }^{139}$, Y.-M. Kim ${ }^{140}$, C. Kimball ${ }^{59}$, E. J. King ${ }^{55}$, P. J. King ${ }^{45}$, M. Kinley-Hanlon ${ }^{127}$, R. Kirchhoff ${ }^{8,9}$, J. S. Kissel ${ }^{45}$, L. Kleybolte ${ }^{141}$, J. H. Klika ${ }^{23}$, S. Klimenko ${ }^{48}$, T. D. Knowles ${ }^{39}$, P. Koch ${ }^{8,9}$, S. M. Koehlenbeck ${ }^{8,9}$, G. Koekoek ${ }^{36,142}$, S. Koley ${ }^{36}$, V. Kondrashov ${ }^{1}$, A. Kontos ${ }^{12}$, N. Koper ${ }^{8,9}$, M. Korobko 141 , W. Z. Korth ${ }^{1}$, I. Kowalska ${ }^{74}$, D. B. Kozak ${ }^{1}$, V. Kringel ${ }^{8,9}$, N. Krishnendu ${ }^{143}$, A. Królak ${ }^{144,145}$, G. Kuehn ${ }^{8,9}$, A. Kumar ${ }^{131}$, P. Kumar ${ }^{146}$, R. Kumar ${ }^{107}$, S. Kumarr ${ }^{16}$, L. Kuo ${ }^{88}$, A. Kutynia ${ }^{144}$, S. Kwang ${ }^{23}$, B. D. Lackey ${ }^{35}$, K. H. Lai ${ }^{91}$, T. L. Lam ${ }^{91}$, M. Landry ${ }^{45}$, B. B. Lane ${ }^{12}$, R. N. Lang ${ }^{147}$, J. Lange ${ }^{58}$, B. Lantz ${ }^{49}$, R. K. Lanza ${ }^{12}$, A. Lartaux-Vollard ${ }^{25}$, P. D. Lasky ${ }^{6}$, M. Laxen ${ }^{7}$, A. Lazzarini ${ }^{1}$, C. Lazzaro ${ }^{52}$, P. Leaci ${ }^{31,113}$, S. Leavey,9, Y. K. Lecoeuche ${ }^{45}$, C. H. Lee ${ }^{93}$, H. K. Lee ${ }^{148^{\prime}}$, H. M. Lee ${ }^{149}$, H. W. Lee ${ }^{138}$, J. Lee ${ }^{92}$, K. Lee ${ }^{44}$, J. Lehmann ${ }^{8,9}$, A. Lenon ${ }^{39}$, N. Leroy ${ }^{25}$, N. Letendre ${ }^{32}$, Y. Levin ${ }^{6,98}$, J. Li $^{83}$,

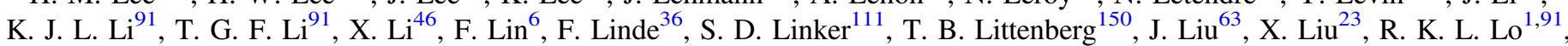
N. A. Lockerbie ${ }^{24}$, L. T. London ${ }^{68}$, A. Longo ${ }^{151,152}$, M. Lorenzini ${ }^{14,15}$, V. Loriette ${ }^{153}$, M. Lormand ${ }^{7}$, G. Losurdo ${ }^{19}$, J. D. Lough ${ }^{8,9}$, C. O. Lousto ${ }^{58}$, G. Lovelace ${ }^{26}$, M. E. Lower ${ }^{38}$, H. Lück ${ }^{8,9}$, D. Lumaca ${ }^{29,30}$, A. P. Lundgren ${ }^{154}$, R. Lynch ${ }^{12}$, Y. Ma ${ }^{46}$, R. Macas ${ }^{68}$, S. Macfoy ${ }^{24}$, M. MacInnis ${ }^{12}$, D. M. Macleod ${ }^{68}$, A. Macquet ${ }^{65}$, F. Magaña-Sandoval ${ }^{42}$, L. Magaña Zertuche ${ }^{85}$, R. M. Magee ${ }^{87}$, E. Majorana ${ }^{31}$, I. Maksimovic ${ }^{153}$, A. Malik ${ }^{61}$, N. Man ${ }^{65}$, V. Mandic ${ }^{43}$, V. Mangano ${ }^{44}$, G. L. Mansell ${ }^{12,45}$, M. Manske ${ }^{21,23}$, M. Mantovani ${ }^{28}$, F. Marchesoni ${ }^{41,50}$, F. Marion ${ }^{32}$, S. Márka ${ }^{98}$, Z. Márka ${ }^{98}$, C. Markakis ${ }^{10,17}$, A. S. Markosyan ${ }^{49}$, A. Markowitz ${ }^{1}$, E. Maros ${ }^{1}$, A. Marquina ${ }^{102}$, S. Marsat ${ }^{35}$, F. Martelli ${ }^{72,73}$, I. W. Martin ${ }^{44}$, R. M. Martin 34 , D. V. Martynov ${ }^{11}$, K. Mason ${ }^{12}$, E. Massera $^{108}$, A. Masserot ${ }^{32}$, T. J. Massinger ${ }^{1}$, M. Masso-Reid ${ }^{44}$, S. Mastrogiovanni ${ }^{31,113}$, A. Matas ${ }^{35,43}$, F. Matichard ${ }^{1,12}$, L. Matone ${ }^{98}$, N. Mavalvala ${ }^{12}$, N. Mazumder ${ }^{69}$, J. J. McCann ${ }^{63}$, R. McCarthy ${ }^{45}$, D. E. McClelland ${ }^{21}$, S. McCormick ${ }^{7}$, L. McCuller ${ }^{12}$, S. C. McGuire ${ }^{155}$, J. McIver ${ }^{1}$, D. J. McManus ${ }^{21}$, T. McRae ${ }^{21}$, S. T. McWilliams ${ }^{39}$, D. Meacher ${ }^{87}$, G. D. Meadors ${ }^{6}$, M. Mehmet ${ }^{8,9}$, A. K. Mehta ${ }^{16}$, J. Meidam ${ }^{36}$, A. Melatos ${ }^{97}$, G. Mendell ${ }^{45}$, R. A. Mercer ${ }^{23}$, L. Mereni ${ }^{22}$, E. L. Merilh ${ }^{45}$, M. Merzougui ${ }^{65}$, S. Meshkov ${ }^{1}$, C. Messenger ${ }^{44}$, C. Messick ${ }^{87}$, R. Metzdorff ${ }^{71}$, P. M. Meyers ${ }^{97}$, H. Miao ${ }^{11}$, C. Michel ${ }^{22}$, H. Middleton ${ }^{97}$, E. E. Mikhailov ${ }^{156}$, L. Milano ${ }^{5,79}$, A. L. Miller ${ }^{48}$, A. Miller ${ }^{31,113}$, M. Millhouse ${ }^{53}$, J. C. Mills ${ }^{68}$, M. C. Milovich-Goff ${ }^{111}$, O. Minazzoli ${ }^{65,157}$, Y. Minenkov ${ }^{30}$, A. Mishkin ${ }^{48}$, C. Mishra ${ }^{158}$, T. Mistry ${ }^{108}$, S. Mitra ${ }^{3}$, V. P. Mitrofanov ${ }^{62}$, G. Mitselmakher $^{48}$, R. Mittleman ${ }^{12}$, G. Mo ${ }^{94}$, D. Moffa ${ }^{115}$, K. Mogushi ${ }^{85}$, S. R. P. Mohapatra ${ }^{12}$, M. Montani ${ }^{72,73}$, C. J. Moore ${ }^{10}$, D. Moraru ${ }^{45}$, G. Moreno $^{45}$, S. Morisaki ${ }^{82}$, B. Mours ${ }^{32}$, C. M. Mow-Lowry ${ }^{11}$, Arunava Mukherjee ${ }^{8,9}$, D. Mukherjee ${ }^{23}$, S. Mukherjee ${ }^{104}$, N. Mukund ${ }^{3}$, A. Mullavey ${ }^{7}$, J. Munch ${ }^{55}$, E. A. Muñiz ${ }^{42}$, M. Muratore ${ }^{33}$, P. G. Murray ${ }^{44}$, A. Nagar ${ }^{86,159,160}$, I. Nardecchia ${ }^{29,30}$, L. Naticchioni ${ }^{31,113}$, R. K. Nayak ${ }^{161}$, J. Neilson ${ }^{111}$, G. Nelemans ${ }^{36,64}$, T. J. N. Nelson ${ }^{7}$, M. Nery ${ }^{8,9}$, A. Neunzert ${ }^{126}$, K. Y. Ng $^{12}$, S. Ng ${ }^{55}$, P. Nguyen ${ }^{70}$, D. Nichols ${ }^{36,128}$, S. Nissanke ${ }^{36,128}$, F. Nocera $^{28}$, C. North $^{68}$, L. K. Nuttall ${ }^{154}$, M. Obergaulinger ${ }^{20}$, J. Oberling ${ }^{45}$, B. D. O’Brien ${ }^{48}$, G. D. O’Dea ${ }^{11}$, G. H. Ogin ${ }^{162}$, J. J. Oh ${ }^{139}$, S. H. Oh ${ }^{139}$, F. Ohme ${ }^{89}$, H. Ohta ${ }^{82}$, M. A. Okada ${ }^{13}$, M. Oliver ${ }^{99}$, P. Oppermann ${ }^{8,9}$, Richard J. Oram ${ }^{7}$, B. O'Reilly ${ }^{7}$, R. G. Ormiston ${ }^{43}$, L. F. Ortega ${ }^{48}$, R. O'Shaughnessy ${ }^{58}$,

S. Ossokine ${ }^{35}$, D. J. Ottaway ${ }^{55}$, H. Overmier ${ }^{7}$, B. J. Owen ${ }^{84}$, A. E. Pace ${ }^{87}$, G. Pagano ${ }^{18,19}$, M. A. Page ${ }^{63}$, A. Pai ${ }^{122}$, S. A. Pai ${ }^{61}$, J. R. Palamos ${ }^{70}$, O. Palashov ${ }^{136}$, C. Palomba ${ }^{31}$, A. Pal-Singh ${ }^{141}$, Huang-Wei Pan ${ }^{88}$, B. Pang ${ }^{46}$, P. T. H. Pang ${ }^{91}$, C. Pankow ${ }^{59}$, F. Pannarale ${ }^{31,113}$, B. C. Pant ${ }^{61}$, F. Paoletti ${ }^{19}$, A. Paoli ${ }^{28}$, A. Parida ${ }^{3}$, W. Parker ${ }^{7,155}$, D. Pascucci ${ }^{44}$, A. Pasqualetti ${ }^{28}$,

R. Passaquieti ${ }^{18,19}$, D. Passuello ${ }^{19}$, M. Patil ${ }^{145}$, B. Patricelli ${ }^{18,19}$, B. L. Pearlstone ${ }^{44}$, C. Pedersen ${ }^{68}$, M. Pedraza ${ }^{1}$, R. Pedurand ${ }^{22,163}$, A. Pele ${ }^{7}$, S. Penn ${ }^{164}$, C. J. Perez ${ }^{45}$, A. Perreca ${ }^{96,112}$, H. P. Pfeiffer ${ }^{35,118}$, M. Phelps ${ }^{8,9}$, K. S. Phukon ${ }^{3}$, O. J. Piccinni ${ }^{31,113}$, M. Pichot ${ }^{65}$, F. Piergiovanni ${ }^{72,73}$, G. Pillant ${ }^{28}$, L. Pinard ${ }^{22}$, M. Pirello ${ }^{45}$, M. Pitkin ${ }^{44}$, R. Poggiani ${ }^{18,19}$, D. Y. T. Pong ${ }^{91}$,

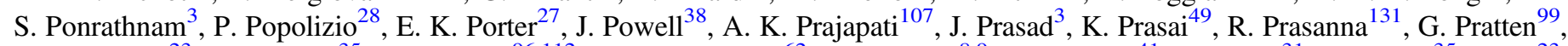
T. Prestegard ${ }^{23}$, S. Privitera ${ }^{35}$, G. A. Prodi ${ }^{96,112}$, L. G. Prokhorov ${ }^{62}$, O. Puncken ${ }^{8,9}$, M. Punturo ${ }^{41}$, P. Puppo ${ }^{31}$, M. Pürrer $^{35}$, H. Qi $^{23}$, V. Quetschke ${ }^{104}$, P. J. Quinonez ${ }^{33}$, E. A. Quintero ${ }^{1}$, R. Quitzow-James ${ }^{70}$, F. J. Raab ${ }^{45}$, H. Radkins ${ }^{45}$, N. Radulescu ${ }^{65}$, P. Raffai ${ }^{106}$, S. Raja ${ }^{61}$, C. Rajan ${ }^{61}$, B. Rajbhandari ${ }^{84}$, M. Rakhmanov ${ }^{104}$, K. E. Ramirez ${ }^{104}$, A. Ramos-Buades ${ }^{99}$, Javed Rana ${ }^{3}$, K. Rao ${ }^{59}$, P. Rapagnani ${ }^{31,113}$, V. Raymond ${ }^{68}$, M. Razzano ${ }^{18,19}$, J. Read ${ }^{26}$, T. Regimbau ${ }^{32}$, L. Rei $^{60}$, S. Reid $^{24}$, D. H. Reitze ${ }^{1,48}$, W. Ren ${ }^{17}$, F. Ricci ${ }^{31,113}$, C. J. Richardson ${ }^{33}$, J. W. Richardson ${ }^{1}$, P. M. Ricker ${ }^{17}$, K. Riles ${ }^{126}$, M. Rizzo ${ }^{59}$, N. A. Robertson ${ }^{1,44}$, R. Robie ${ }^{44}$, F. Robinet ${ }^{25}$, A. Rocchi ${ }^{30}$, L. Rolland ${ }^{32}$, J. G. Rollinss ${ }^{1}$, V. J. Roma ${ }^{70}$, M. Romanelli ${ }^{67}$, R. Romano ${ }^{4,5}$, C. L. Romel ${ }^{45}$, J. H. Romie ${ }^{7}$, K. Rose $^{115}$, D. Rosińska ${ }^{54,165}$, S. G. Rosofsky ${ }^{17}$, M. P. Ross $^{166}$, S. Rowan $^{44}$, A. Rüdiger ${ }^{8,9,193}$, P. Ruggi ${ }^{28}$, G. Rutins ${ }^{167}$, K. Ryan ${ }^{45}$, S. Sachdev ${ }^{1}$, T. Sadecki ${ }^{45}$, M. Sakellariadou ${ }^{133}$, L. Salconi ${ }^{28}$, M. Saleem ${ }^{143}$, A. Samajdar ${ }^{36}$, L. Sammut ${ }^{6}$, E. J. Sanchez ${ }^{1}$, L. E. Sanchez ${ }^{1}$, N. Sanchis-Gual ${ }^{20}$, V. Sandberg ${ }^{45}$, J. R. Sanders ${ }^{42}$, K. A. Santiago ${ }^{34}$, N. Sarin ${ }^{6}$, B. Sassolas ${ }^{22}$, P. R. Saulson ${ }^{42}$, 
O. Sauter ${ }^{126}$, R. L. Savage ${ }^{45}$, P. Schale ${ }^{70}$, M. Scheel ${ }^{46}$, J. Scheuer ${ }^{59}$, P. Schmidt ${ }^{64}$, R. Schnabel ${ }^{141}$, R. M. S. Schofield ${ }^{70}$, A. Schönbeck ${ }^{141}$, E. Schreiber ${ }^{8,9}$, B. W. Schulte ${ }^{8,9}$, B. F. Schutz ${ }^{68}$, S. G. Schwalbe ${ }^{33}$, J. Scott ${ }^{44}$, S. M. Scott ${ }^{21}$, E. Seidel ${ }^{17}$, D. Sellers ${ }^{7}$, A. S. Sengupta ${ }^{168}$, N. Sennett ${ }^{35}$, D. Sentenac ${ }^{28}$, V. Sequino ${ }^{14,29,30}$, A. Sergeev ${ }^{136}$, Y. Setyawati ${ }^{8,9}$, D. A. Shaddock ${ }^{21}$, T. Shaffer ${ }^{45}$, M. S. Shahriar ${ }^{59}$, M. B. Shaner ${ }^{11}$, L. Shao ${ }^{35}$, P. Sharma ${ }^{61}$, P. Shawhan ${ }^{76}$, H. Shen ${ }^{17}$, R. Shink ${ }^{169}$, D. H. Shoemaker ${ }^{12}$, D. M. Shoemaker ${ }^{77}$, S. ShyamSundar ${ }^{61}$, K. Siellez ${ }^{77}$, M. Sieniawska ${ }^{54}$, D. Sigg ${ }^{45}$, A. D. Silva ${ }^{13}$, L. P. Singer ${ }^{80}$, N. Singh ${ }^{74}$, A. Singhal ${ }^{14,31}$, A. M. Sintes ${ }^{99}$, S. Sitmukhambetov ${ }^{104}$, V. Skliris ${ }^{68}$, B. J. J. Slagmolen ${ }^{21}$, T. J. Slaven-Blair ${ }^{63}$, J. R. Smith ${ }^{26}$, R. J. E. Smith ${ }^{6}$, S. Somala ${ }^{170}$, E. J. Son ${ }^{139}$, B. Sorazu ${ }^{44}$, F. Sorrentino ${ }^{60}$, T. Souradeep ${ }^{3}$, E. Sowell ${ }^{84}$, A. P. Spencer ${ }^{44}$, A. K. Srivastava ${ }^{107}$, V. Srivastava ${ }^{42}$, K. Staats ${ }^{59}$, C. Stachie $^{65}$, M. Standke ${ }^{8,9}$, D. A. Steer ${ }^{27}$, M. Steinke ${ }^{8,9}$, J. Steinlechner ${ }^{44,141}$, S. Steinlechner ${ }^{141}$, D. Steinmeyer ${ }^{8,9}$, S. P. Stevenson ${ }^{38}$, D. Stocks ${ }^{49}$, R. Stone ${ }^{104}$, D. J. Stops ${ }^{11}$, K. A. Strain ${ }^{44}$, G. Stratta ${ }^{72,73}$, S. E. Strigin ${ }^{62}$, A. Strunk ${ }^{45}$, R. Sturani ${ }^{171}$, A. L. Stuver ${ }^{172}$, V. Sudhir ${ }^{12}$, T. Z. Summerscales ${ }^{173}$, L. Sun ${ }^{1}$, S. Sunil ${ }^{107}$, J. Suresh $^{3}$, P. J. Sutton ${ }^{68}$, B. L. Swinkels ${ }^{36}$, M. J. Szczepańczyk ${ }^{33}$, M. Tacca ${ }^{36}$, S. C. Tait ${ }^{44}$, C. Talbot ${ }^{6}$, D. Talukder ${ }^{70}$, D. B. Tanner ${ }^{48}$, M. Tápai ${ }^{123}$, A. Taracchini ${ }^{35}$, J. D. Tasson ${ }^{94}$, R. Taylor ${ }^{1}$, F. Thies ${ }^{8,9}$, M. Thomas ${ }^{7}$, P. Thomas ${ }^{45}$, S. R. Thondapu ${ }^{61}$, K. A. Thorne ${ }^{7}$, E. Thrane ${ }^{6}$, Shubhanshu Tiwari ${ }^{96,112}$, Srishti Tiwari ${ }^{124}$, V. Tiwari ${ }^{68}$, K. Toland $^{44}$, M. Tonelli ${ }^{18,19}$, Z. Tornasi $^{44}$, A. Torres-Forné ${ }^{174}$, C. I. Torrie ${ }^{1}$, D. Töyrä ${ }^{11}$, F. Travasso ${ }^{28,41}$, G. Traylor ${ }^{7}$, M. C. Tringali ${ }^{74}$, A. Trovato ${ }^{27}$, L. Trozzo ${ }^{19,175}$, R. Trudeau ${ }^{1}$, K. W. Tsang ${ }^{36}$, M. Tse ${ }^{12}$, R. Tso ${ }^{46}$, L. Tsukada ${ }^{82}$, D. Tsuna ${ }^{82}$, D. Tuyenbayev ${ }^{104}$, K. Ueno ${ }^{82}$, D. Ugolini ${ }^{176}$, C. S. Unnikrishnan ${ }^{124}$, A. L. Urban ${ }^{2}$, S. A. Usman ${ }^{68}$, H. Vahlbruch ${ }^{9}$, G. Vajente ${ }^{1}$, G. Valdes $^{2}$, N. van Bakel ${ }^{36}$, M. van Beuzekom ${ }^{36}$, J. F. J. van den Brand ${ }^{36,75}$,

C. Van Den Broeck ${ }^{36,177}$, D. C. Vander-Hyde ${ }^{42}$, J. V. van Heijningen ${ }^{63}$, L. van der Schaaf ${ }^{36}$, A. A. van Veggel ${ }^{44}$, M. Vardaro $^{51,52}$, V. Varma ${ }^{46}$, S. Vass ${ }^{1}$, M. Vasúth ${ }^{47}$, A. Vecchio ${ }^{11}$, G. Vedovato ${ }^{52}$, J. Veitch ${ }^{44}$, P. J. Veitch ${ }^{55}$, K. Venkateswara ${ }^{166}$, G. Venugopalan ${ }^{1}$, D. Verkindt ${ }^{32}$, F. Vetrano ${ }^{72,73}$, A. Viceré ${ }^{72,73}$, A. D. Viets ${ }^{23}$, D. J. Vine ${ }^{167}$, J.-Y. Vinet $^{65}$, S. Vitale $^{12}$, T. Vo ${ }^{42}$, H. Vocca $^{40,41}$, C. Vorvick ${ }^{45}$, S. P. Vyatchanin ${ }^{62}$, A. R. Wade ${ }^{1}$, L. E. Wade ${ }^{115}$, M. Wade ${ }^{115}$, R. Walet ${ }^{36}$, M. Walker ${ }^{26}$, L. Wallace ${ }^{1}$, S. Walsh $^{23}$, G. Wang ${ }^{14,19}$, H. Wang ${ }^{11}$, J. Z. Wang ${ }^{126}$, W. H. Wang ${ }^{104}$, Y. F. Wang ${ }^{91}$, R. L. Ward ${ }^{21}$, Z. A. Warden ${ }^{33}$, J. Warner ${ }^{45}$, M. Was ${ }^{32}$, J. Watchi ${ }^{100}$, B. Weaver ${ }^{45}$, L.-W. Wei ${ }^{8,9}$, M. Weinert ${ }^{8,9}$, A. J. Weinstein ${ }^{1}$, R. Weiss ${ }^{12}$, F. Wellmann ${ }^{8,9}$, L. Wen $^{63}$, E. K. Wessel ${ }^{17}$, P. Weßels ${ }^{8,9}$, J. W. Westhouse ${ }^{33}$, K. Wette ${ }^{21}$, J. T. Whelan ${ }^{58}$, B. F. Whiting ${ }^{48}$, C. Whittle ${ }^{12}$, D. M. Wilken ${ }^{8,9}$, D. Williams ${ }^{44}$, A. R. Williamson ${ }^{36,128}$, J. L. Willis ${ }^{1}$, B. Willke ${ }^{8,9}$, M. H. Wimmer ${ }^{8,9}$, W. Winkler ${ }^{8,9}$, C. C. Wipf ${ }^{1}$, H. Wittel ${ }^{8,9}$, G. Woan ${ }^{44}$, J. Woehler ${ }^{8,9}$, J. K. Wofford ${ }^{58}$, J. Worden ${ }^{45}$, J. L. Wright ${ }^{44}$, D. S. Wu ${ }^{8,9}$, D. M. Wysocki ${ }^{58}$, L. Xiao ${ }^{1}$, H. Yamamoto ${ }^{1}$,

C. C. Yancey ${ }^{76}$, L. Yang ${ }^{114}$, M. J. Yapp ${ }^{21}$, M. Yazback ${ }^{48}$, D. W. Yeeles ${ }^{68}$, Hang Yu ${ }^{12}$, Haocun Yu ${ }^{12}$, S. H. R. Yuen ${ }^{91}$, M. Yvert ${ }^{32}$, A. K. Zadrożny ${ }^{104,144}$, M. Zanolin ${ }^{33}$, T. Zelenova ${ }^{28}$, J.-P. Zendri ${ }^{52}$, M. Zevin ${ }^{59}$, J. Zhang ${ }^{63}$, L. Zhang ${ }^{1}$, T. Zhang ${ }^{44}$, C. Zhao ${ }^{63}$, M. Zhou ${ }^{59}$, Z. Zhou ${ }^{59}$, X. J. Zhu ${ }^{6}$, M. E. Zucker ${ }^{1,12}$, J. Zweizig ${ }^{1}$

The LIGO Scientific Collaboration and the Virgo Collaboration,

Z. Arzoumanian ${ }^{178}$, S. Bogdanov ${ }^{179}$, I. Cognard ${ }^{180,181}$, A. Corongiu ${ }^{182}$, T. Enoto $^{183}$, P. Freire ${ }^{184}$, K. C. Gendreau ${ }^{178}$,

L. Guillemot ${ }^{180,181}$, A. K. Harding ${ }^{185}$, F. Jankowski ${ }^{186}$, M. J. Keith ${ }^{186}$, M. Kerr ${ }^{187}$, A. Lyne ${ }^{186}$, J. Palfreyman ${ }^{188}$, A. Possenti ${ }^{\text {182,189, }}{ }^{\text {, }}$ A. Ridolff ${ }^{184}$, B. Stappers ${ }^{186}$, G. Theureau ${ }^{180,181,190}$, and P. Weltervrede ${ }^{186}$

${ }^{1}$ LIGO, California Institute of Technology, Pasadena, CA 91125, USA

${ }^{2}$ Louisiana State University, Baton Rouge, LA 70803, USA

${ }^{3}$ Inter-University Centre for Astronomy and Astrophysics, Pune 411007, India

${ }^{4}$ Università di Salerno, Fisciano, I-84084 Salerno, Italy

${ }^{5}$ INFN, Sezione di Napoli, Complesso Universitario di Monte S.Angelo, I-80126 Napoli, Italy

${ }^{6}$ OzGrav, School of Physics \& Astronomy, Monash University, Clayton 3800, Victoria, Australia

${ }^{7}$ LIGO Livingston Observatory, Livingston, LA 70754, USA

${ }^{8}$ Max Planck Institute for Gravitational Physics (Albert Einstein Institute), D-30167 Hannover, Germany

${ }^{9}$ Leibniz Universität Hannover, D-30167 Hannover, Germany

${ }^{10}$ University of Cambridge, Cambridge CB2 1TN, UK

${ }^{11}$ University of Birmingham, Birmingham B15 2TT, UK

${ }^{12}$ LIGO, Massachusetts Institute of Technology, Cambridge, MA 02139, USA

${ }^{13}$ Instituto Nacional de Pesquisas Espaciais, 12227-010 Sao José dos Campos, Sao Paulo, Brazil

${ }^{14}$ Gran Sasso Science Institute (GSSI), I-67100 L'Aquila, Italy

${ }^{15}$ INFN, Laboratori Nazionali del Gran Sasso, I-67100 Assergi, Italy

${ }^{16}$ International Centre for Theoretical Sciences, Tata Institute of Fundamental Research, Bengaluru 560089, India

${ }^{17}$ NCSA, University of Illinois at Urbana-Champaign, Urbana, IL 61801, USA

${ }^{18}$ Università di Pisa, I-56127 Pisa, Italy

${ }^{19}$ INFN, Sezione di Pisa, I-56127 Pisa, Italy

${ }^{20}$ Departamento de Astronomía y Astrofísica, Universitat de València, E-46100 Burjassot, València, Spain

${ }_{21}^{21}$ OzGrav, Australian National University, Canberra, Australian Capital Territory 0200, Australia

${ }^{22}$ Laboratoire des Matériaux Avancés (LMA), CNRS/IN2P3, F-69622 Villeurbanne, France

${ }^{23}$ University of Wisconsin-Milwaukee, Milwaukee, WI 53201, USA

${ }^{24}$ SUPA, University of Strathclyde, Glasgow G1 1XQ, UK

${ }^{25}$ LAL, Univ. Paris-Sud, CNRS/IN2P3, Université Paris-Saclay, F-91898 Orsay, France

${ }^{26}$ California State University Fullerton, Fullerton, CA 92831, USA

${ }^{27}$ APC, AstroParticule et Cosmologie, Université Paris Diderot, CNRS/IN2P3, CEA/Irfu, Observatoire de Paris, Sorbonne Paris Cité, F-75205 Paris Cedex 13, France

${ }^{28}$ European Gravitational Observatory (EGO), I-56021 Cascina, Pisa, Italy

${ }^{29}$ Università di Roma Tor Vergata, I-00133 Roma, Italy

${ }^{30}$ INFN, Sezione di Roma Tor Vergata, I-00133 Roma, Italy

${ }^{31}$ INFN, Sezione di Roma, I-00185 Roma, Italy

${ }^{32}$ Laboratoire d'Annecy de Physique des Particules (LAPP), Univ. Grenoble Alpes, Université Savoie Mont Blanc, CNRS/IN2P3, F-74941 Annecy, France

${ }^{33}$ Embry-Riddle Aeronautical University, Prescott, AZ 86301, USA 


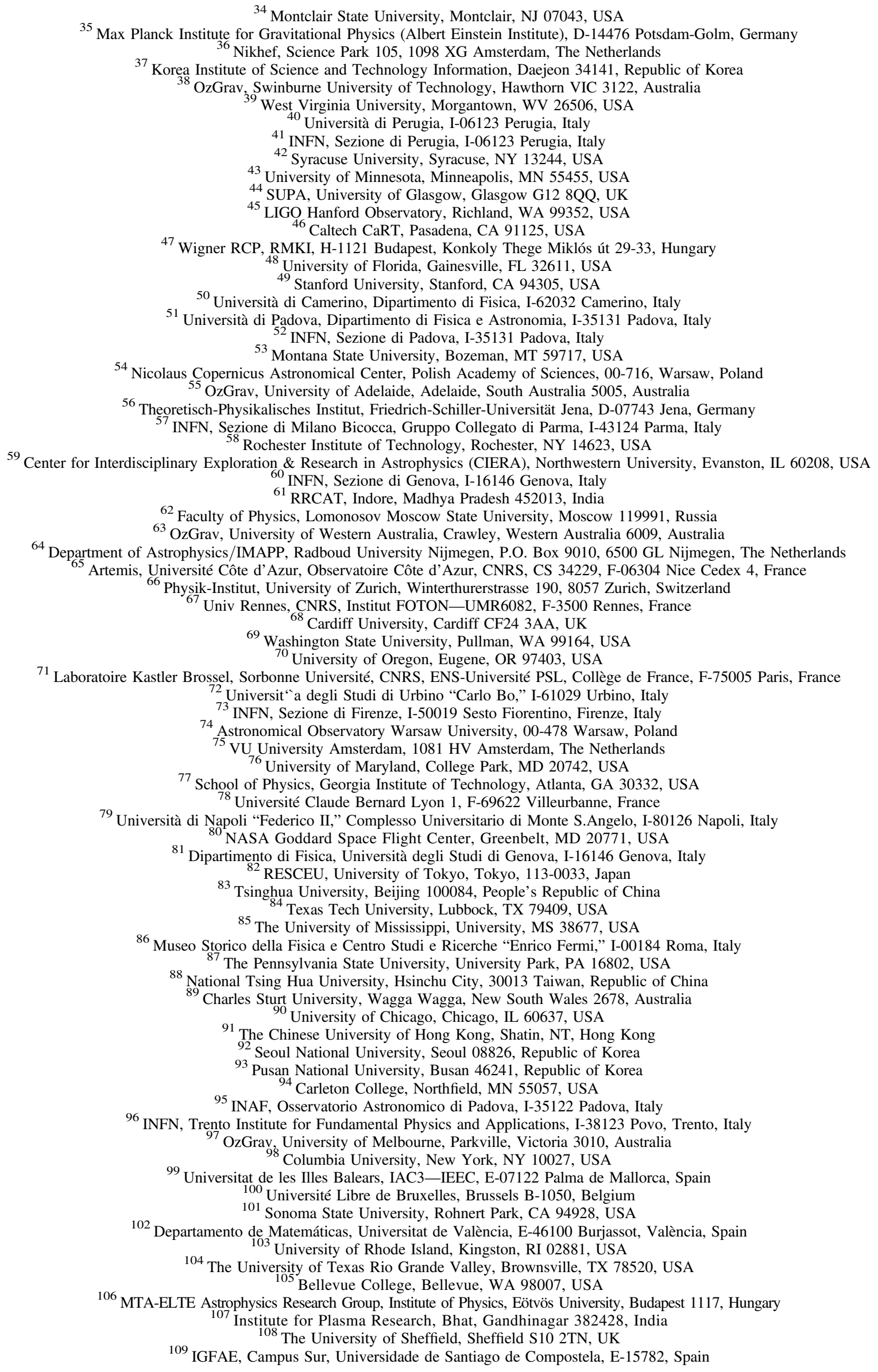




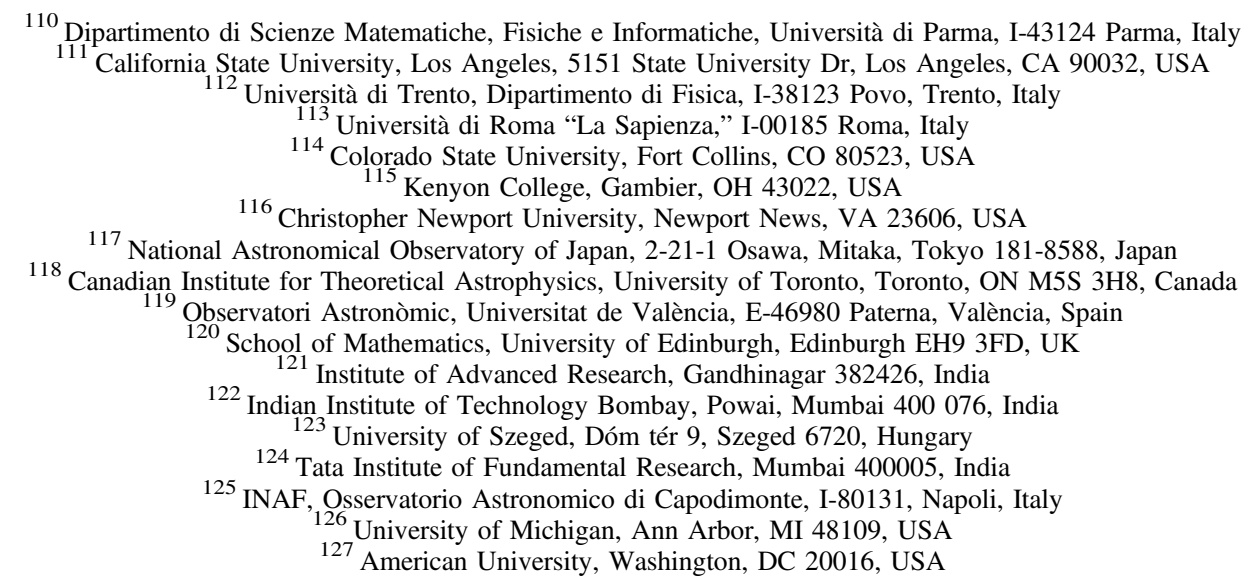

${ }^{128}$ GRAPPA, Anton Pannekoek Institute for Astronomy and Institute of High-Energy Physics, University of Amsterdam, Science Park 904, 1098 XH Amsterdam, The Netherlands

${ }^{129}$ Delta Institute for Theoretical Physics, Science Park 904, 1090 GL Amsterdam, The Netherlands

${ }^{130}$ Department of Physics and Astronomy, Haverford College, 370 Lancaster Avenue, Haverford, PA 19041, USA

${ }^{131}$ Directorate of Construction, Services \& Estate Management, Mumbai 400094, India

${ }^{132}$ University of Białystok, 15-424 Białystok, Poland

${ }^{133}$ King's College London, University of London, London WC2R 2LS, UK

${ }^{134}$ University of Southampton, Southampton SO17 1BJ, UK

${ }^{135}$ University of Washington Bothell, Bothell, WA 98011, USA

${ }^{136}$ Institute of Applied Physics, Nizhny Novgorod, 603950, Russia

${ }^{137}$ Ewha Womans University, Seoul 03760, Republic of Korea

${ }^{138}$ Inje University Gimhae, South Gyeongsang 50834, Republic of Korea

${ }^{139}$ National Institute for Mathematical Sciences, Daejeon 34047, Republic of Korea

${ }^{140}$ Ulsan National Institute of Science and Technology, Ulsan 44919, Republic of Korea

${ }^{141}$ Universität Hamburg, D-22761 Hamburg, Germany

${ }^{142}$ Maastricht University, P.O. Box 616, 6200 MD Maastricht, The Netherlands

${ }^{143}$ Chennai Mathematical Institute, Chennai 603103, India

${ }^{144}$ NCBJ, 05-400 Świerk-Otwock, Poland

${ }^{145}$ Institute of Mathematics, Polish Academy of Sciences, 00656 Warsaw, Poland

${ }_{146}$ Cornell University, Ithaca, NY 14850 , USA

${ }^{147}$ Hillsdale College, Hillsdale, MI 49242, USA

${ }^{148}$ Hanyang University, Seoul 04763 , Republic of Korea

${ }^{149}$ Korea Astronomy and Space Science Institute, Daejeon 34055, Republic of Korea

${ }^{150}$ NASA Marshall Space Flight Center, Huntsville, AL 35811, USA

${ }^{151}$ Dipartimento di Matematica e Fisica, Università degli Studi Roma Tre, I-00146 Roma, Italy

${ }^{152}$ INFN, Sezione di Roma Tre, I-00146 Roma, Italy ${ }_{153}$ ESPCI, CNRS, F-75005 Paris, France

${ }^{154}$ University of Portsmouth, Portsmouth, PO1 3FX, UK

${ }^{155}$ Southern University and A\&M College, Baton Rouge, LA 70813, USA

${ }^{156}$ College of William and Mary, Williamsburg, VA 23187, USA

${ }^{157}$ Centre Scientifique de Monaco, 8 quai Antoine Ier, MC-98000, Monaco

${ }_{159}^{158}$ Indian Institute of Technology Madras, Chennai 600036, India

${ }^{159}$ INFN Sezione di Torino, Via P. Giuria 1, I-10125 Torino, Italy

${ }^{160}$ Institut des Hautes Etudes Scientifiques, F-91440 Bures-sur-Yvette, France

${ }^{162}{ }^{161}$ IISER-Kolkata, Mohanpur, West Bengal 741252, India

${ }^{162}$ Whitman College, 345 Boyer Avenue, Walla Walla, WA 99362, USA

${ }^{163}$ Université de Lyon, F-69361 Lyon, France

${ }^{164}$ Hobart and William Smith Colleges, Geneva, NY 14456, USA

165 Janusz Gil Institute of Astronomy, University of Zielona Góra, 65-265 Zielona Góra, Poland

${ }^{166}$ University of Washington, Seattle, WA 98195, USA

${ }^{167}$ SUPA, University of the West of Scotland, Paisley PA1 2BE, UK

${ }^{168}$ Indian Institute of Technology, Gandhinagar Ahmedabad Gujarat 382424, India

${ }_{169}$ Université de Montréal/Polytechnique, Montreal, QC H3T 1J4, Canada

${ }^{170}$ Indian Institute of Technology Hyderabad, Sangareddy, Khandi, Telangana 502285, India

${ }^{171}$ International Institute of Physics, Universidade Federal do Rio Grande do Norte, Natal RN 59078-970, Brazil

${ }^{172}$ Villanova University, 800 Lancaster Ave., Villanova, PA 19085, USA

173 Andrews University, Berrien Springs, MI 49104, USA

${ }^{174}$ Max Planck Institute for Gravitationalphysik (Albert Einstein Institute), D-14476 Potsdam-Golm, Germany

${ }^{175}$ Università di Siena, I-53100 Siena, Italy

${ }^{176}$ Trinity University, San Antonio, TX 78212, USA

${ }^{177}$ Van Swinderen Institute for Particle Physics and Gravity, University of Groningen, Nijenborgh 4, 9747 AG Groningen, The Netherlands

${ }^{178}$ X-Ray Astrophysics Laboratory, NASA Goddard Space Flight Center, Greenbelt, MD 20771, USA

${ }^{179}$ Columbia Astrophysics Laboratory, Columbia University, 550 West 120th Street, New York, NY 10027, USA

${ }^{180}$ Laboratoire de Physique et Chimie de l'Environnement et de l'Espace-Université d'Orléans/CNRS, F-45071 Orléans Cedex 02, France

${ }^{181}$ Station de Radioastronomie de Nançay, Observatoire de Paris, CNRS/INSU, F-18330 Nançay, France

${ }^{182}$ INAF-Osservatorio Astronomico di Cagliari, via della Scienza 5, I-09047 Selargius, Italy

${ }^{183}$ Hakubi Center for Advanced Research and Department of Astronomy, Kyoto University, Kyoto 606-8302, Japan

${ }^{184}$ Max-Planck-Institut für Radioastronomie, Auf dem Hügel 69, D-53121 Bonn, Germany 


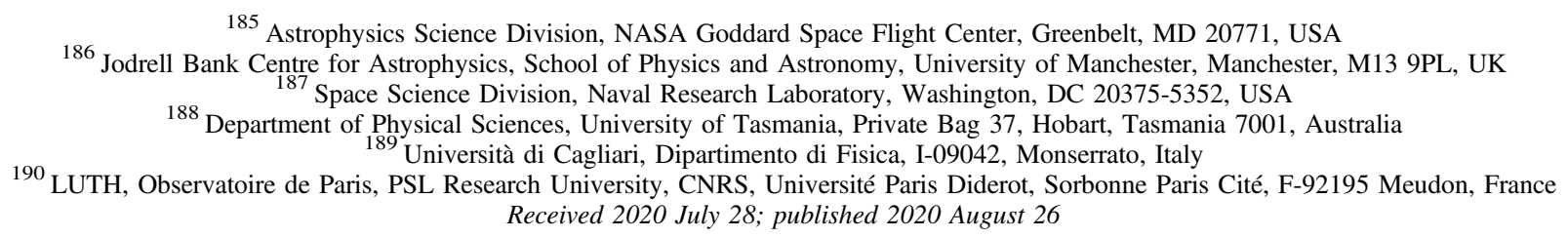

Supporting material: machine-readable table

Two analysis errors have been identified that affect the results for a handful of the high-value pulsars given in Table 1 of Abbott et al. (2019). One affects the Bayesian analysis for the five pulsars that glitched during the analysis period, and the other affects the 5n-vector analysis for J0711-6830. Updated results after correcting the errors are shown in Table 1, which now supersedes the results given for those pulsars in Table 1 of Abbott et al. (2019). Updated versions of figures can be seen in Figures 1-4.

Bayesian analysis.-For the glitching pulsars, the signal phase evolution caused by the glitch was wrongly applied twice and was therefore not consistent with our expected model of the pulsar phase. This error did not affect the $\mathcal{F} / \mathcal{G}$-statistic or $5 n$-vector analysis.

Analyses of the five pulsars PSR J0205+6449, PSR J0534+2200, PSR J0835-4510, PSR J1028-5819, and PSR J1718-3825 have been repeated after correcting for the error. There are small quantitative differences in the results, but the changes do not affect the main conclusions of the paper. The largest differences are for PSR J0835-4510 (the Vela pulsar), for which the updated upper limits from the Bayesian method are found to be between 1.1 and 2 times larger than those obtained when the error was present. This appears primarily to be due to the error leading to the decohering of a strong spectral line in the LIGO Livingston detector and thus lowering the amplitude limit.

$5 n$-vector analysis. - An error was also identified in the settings of the 5n-vector analysis, which affected the upper limit computation at the rotation frequency for $C_{21}^{95 \%}$ of J0711-6830. Specifically, we found an incorrect choice for the range of amplitudes used to inject simulated signals in the $\mathrm{O} 2$ data. The updated upper limit is about 2.5 times worse than that obtained when the error was present. This error did not affect the Bayesian or $\mathcal{F} / \mathcal{G}$-statistic results.

191 Deceased, 2018 February.

192 Deceased, 2017 November.

193 Deceased, 2018 July. 
Table 1

Limits on Gravitational-wave Amplitude and Other Derived Quantities for Five High-value Pulsars from the Three Analysis Methods

\begin{tabular}{|c|c|c|c|c|c|c|c|c|c|c|c|c|c|}
\hline $\begin{array}{l}\text { Pulsar Name } \\
(\mathrm{J} 2000)\end{array}$ & $\begin{array}{c}f_{\text {rot }} \\
(\mathrm{Hz})\end{array}$ & $\begin{array}{c}\dot{P}_{\mathrm{rot}} \\
\left(\mathrm{s} \mathrm{s}^{-1}\right)\end{array}$ & $\begin{array}{l}\text { Distance } \\
(\mathrm{kpc})\end{array}$ & $h_{0}^{\text {sd }}$ & $\begin{array}{l}\text { Analysis } \\
\text { Method }\end{array}$ & $C_{21}^{95 \%}$ & $C_{22}^{95 \%}$ & $h_{0}^{95 \%}$ & $\begin{array}{c}Q_{22}^{95 \%} \\
\left(\mathrm{~kg} \mathrm{~m}^{2}\right)\end{array}$ & $\varepsilon^{95 \%}$ & $h_{0}^{95 \%} / h_{0}^{\text {sd }}$ & $\begin{array}{l}\text { Statistic }^{\mathrm{a}} \\
l=2, m=1,2\end{array}$ & $\begin{array}{c}\text { Statistic }^{\mathrm{b}} \\
l=2, m=2\end{array}$ \\
\hline $\mathrm{J} 0205+6449^{\mathrm{c}}$ & 15.2 & $1.9 \times 10^{-13}$ & 2.00 (c) & $6.9 \times 10^{-25}$ & $\begin{array}{c}\text { Bayesian } \\
\mathcal{F} \text {-statistic } \\
5 n \text {-vector }\end{array}$ & $\begin{array}{c}2.2(1.6) \times 10^{-24} \\
2.2 \times 10^{-24} \\
\ldots\end{array}$ & $\begin{array}{c}2.2(2.9) \times 10^{-26} \\
4.5 \times 10^{-26} \\
\ldots\end{array}$ & $\begin{array}{l}4.5(5.7) \times 10^{-26} \\
8.8 \times 10^{-26} \\
2.9(4.5) \times 10^{-26}\end{array}$ & $\begin{array}{c}7.2(9.0) \times 10^{33} \\
1.4 \times 10^{34} \\
4.6(7.1) \times 10^{33}\end{array}$ & $\begin{array}{c}0.9(1.2) \times 10^{-4} \\
1.8 \times 10^{-4} \\
5.9(9.2) \times 10^{-5}\end{array}$ & $\begin{array}{c}0.065(0.082) \\
0.13 \\
0.042(0.065)\end{array}$ & $\begin{array}{c}-4.8(-4.7) \\
0.71 \\
\ldots\end{array}$ & $\begin{array}{c}-2.8(-2.6) \\
0.26 \\
0.41\end{array}$ \\
\hline $\mathrm{J} 0534+2200^{\mathrm{C}}$ & 29.7 & $4.2 \times 10^{-13}$ & 2.00 & $1.4 \times 10^{-24}$ & $\begin{array}{c}\text { Bayesian } \\
\mathcal{F} \text {-statistic } \\
5 n \text {-vector }\end{array}$ & $\begin{array}{l}8.1(5.9) \times 10^{-26} \\
1.6(1.1) \times 10^{-25} \\
1.7(1.3) \times 10^{-25}\end{array}$ & $\begin{array}{c}8.9(7.6) \times 10^{-27} \\
1.1(1.1) \times 10^{-26} \\
\ldots\end{array}$ & $\begin{array}{l}1.9(1.5) \times 10^{-26} \\
2.2(1.3) \times 10^{-26} \\
2.9(2.9) \times 10^{-26}\end{array}$ & $\begin{array}{l}7.8(6.3) \times 10^{32} \\
9.1(5.4) \times 10^{32} \\
1.2(1.2) \times 10^{33}\end{array}$ & $\begin{array}{l}1.0(0.8) \times 10^{-5} \\
1.2(0.7) \times 10^{-5} \\
1.6(1.6) \times 10^{-5}\end{array}$ & $\begin{array}{c}0.013(0.011) \\
0.015(0.0091) \\
0.02(0.02)\end{array}$ & $\begin{array}{c}-5.2(-5.3) \\
0.32(0.18) \\
0.70\end{array}$ & $\begin{array}{c}-2.6(-2.6) \\
0.65(0.87) \\
0.45\end{array}$ \\
\hline $\mathrm{J} 0711-6830^{\mathrm{c}}$ & 182.1 & $1.4 \times 10^{-20}$ & 0.11 (b) & $1.2 \times 10^{-26}$ & $\begin{array}{c}\text { Bayesian } \\
\mathcal{F} \text {-statistic } \\
5 n \text {-vector }\end{array}$ & $\begin{array}{c}2.6 \times 10^{-26} \\
\cdots \\
3.0 \times 10^{-26}\end{array}$ & $\begin{array}{c}7.0 \times 10^{-27} \\
\ldots \\
\ldots\end{array}$ & $\begin{array}{c}1.5 \times 10^{-26} \\
\cdots \\
1.5 \times 10^{-26}\end{array}$ & $\begin{array}{c}9.3 \times 10^{29} \\
\quad \cdots \\
9.1 \times 10^{29}\end{array}$ & $\begin{array}{c}1.2 \times 10^{-8} \\
\ldots \\
1.2 \times 10^{-8}\end{array}$ & $\begin{array}{l}1.3 \\
\cdots \\
1.3\end{array}$ & $\begin{array}{l}-3.1 \\
\ldots \\
0.79\end{array}$ & $\begin{array}{c}-1.9 \\
\ldots \\
0.39\end{array}$ \\
\hline $\mathrm{J} 0835-4510^{\mathrm{c}}$ & 11.2 & $1.2 \times 10^{-13}$ & 0.29 (j) & $3.3 \times 10^{-24}$ & $\begin{array}{c}\text { Bayesian } \\
\mathcal{F} \text {-statistic } \\
5 n \text {-vector }\end{array}$ & $\begin{array}{c}1.5(1.4) \times 10^{-23} \\
1.3(1.1) \times 10^{-23} \\
\ldots\end{array}$ & $\begin{array}{c}1.3(1.0) \times 10^{-25} \\
1.1(0.9) \times 10^{-25} \\
\ldots\end{array}$ & $\begin{array}{l}2.4(2.1) \times 10^{-25} \\
2.6(2.0) \times 10^{-25} \\
2.3(2.4) \times 10^{-25}\end{array}$ & $\begin{array}{l}1.0(0.9) \times 10^{34} \\
1.1(0.8) \times 10^{34} \\
9.7(9.9) \times 10^{33}\end{array}$ & $\begin{array}{l}1.3(1.1) \times 10^{-4} \\
1.4(1.1) \times 10^{-4} \\
1.3(1.3) \times 10^{-4}\end{array}$ & $\begin{array}{c}0.073(0.062) \\
0.078(0.06) \\
0.07(0.071)\end{array}$ & $\begin{array}{c}-3.3(-3.1) \\
0.75(0.75) \\
\ldots\end{array}$ & $\begin{array}{c}-1.8(-2.1) \\
0.75(0.75) \\
0.41\end{array}$ \\
\hline J1028-5819 & 10.9 & $1.6 \times 10^{-14}$ & 1.42 (b) & $2.4 \times 10^{-25}$ & $\begin{array}{c}\text { Bayesian } \\
\mathcal{F} \text {-statistic } \\
5 n \text {-vector }\end{array}$ & $\begin{array}{c}2.0 \times 10^{-23} \\
\ldots \\
\ldots\end{array}$ & $\begin{array}{c}1.0 \times 10^{-25} \\
\ldots \\
\ldots\end{array}$ & $\begin{array}{c}2.4 \times 10^{-25} \\
\cdots \\
1.9 \times 10^{-25}\end{array}$ & $\begin{array}{c}5.2 \times 10^{34} \\
\ldots \\
4.1 \times 10^{34}\end{array}$ & $\begin{array}{c}6.7 \times 10^{-4} \\
\ldots \\
5.3 \times 10^{-4}\end{array}$ & $\begin{array}{c}1 \\
\ldots \\
0.8\end{array}$ & $\begin{array}{l}-3.8 \\
\ldots \\
\ldots\end{array}$ & $\begin{array}{l}-2.2 \\
\ldots \\
0.40\end{array}$ \\
\hline J1718-3825 & 13.4 & $1.3 \times 10^{-14}$ & 3.49 (b) & $9.7 \times 10^{-26}$ & $\begin{array}{c}\text { Bayesian } \\
\mathcal{F} \text {-statistic } \\
5 n \text {-vector }\end{array}$ & $\begin{array}{c}3.2 \times 10^{-24} \\
\ldots \\
\ldots\end{array}$ & $\begin{array}{c}3.7 \times 10^{-26} \\
\ldots \\
\ldots\end{array}$ & $\begin{array}{c}7.8 \times 10^{-26} \\
\cdots \\
6.5 \times 10^{-26}\end{array}$ & $\begin{array}{c}2.8 \times 10^{34} \\
\cdots \\
2.3 \times 10^{34}\end{array}$ & $\begin{array}{c}3.6 \times 10^{-4} \\
\ldots \\
3.0 \times 10^{-4}\end{array}$ & $\begin{array}{c}0.8 \\
\cdots \\
0.67\end{array}$ & $\begin{array}{c}-5.7 \\
\ldots \\
\ldots\end{array}$ & $\begin{array}{c}-2.5 \\
\ldots \\
0.67\end{array}$ \\
\hline
\end{tabular}

Notes. For references and other notes see Table 2 in Abbott et al. (2019). Values in parentheses are those produced using the restricted orientation priors described in Section 2.2.4 of Abbott et al. (2019).

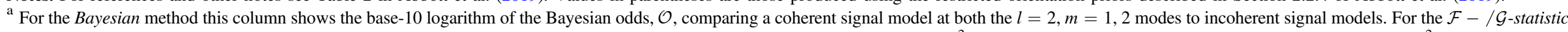

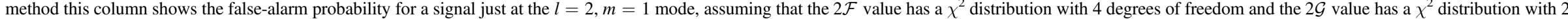

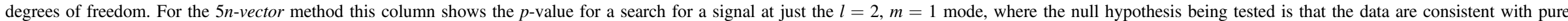
Gaussian noise.

b This is the same as in footnote a, but for all the methods the assumed signal model is from the $l=m=2$ mode.

${ }^{c}$ The observed $\dot{P}$ has been corrected to account for the relative motion between the pulsar and observer.

(This table is available in its entirety in machine-readable form.) 


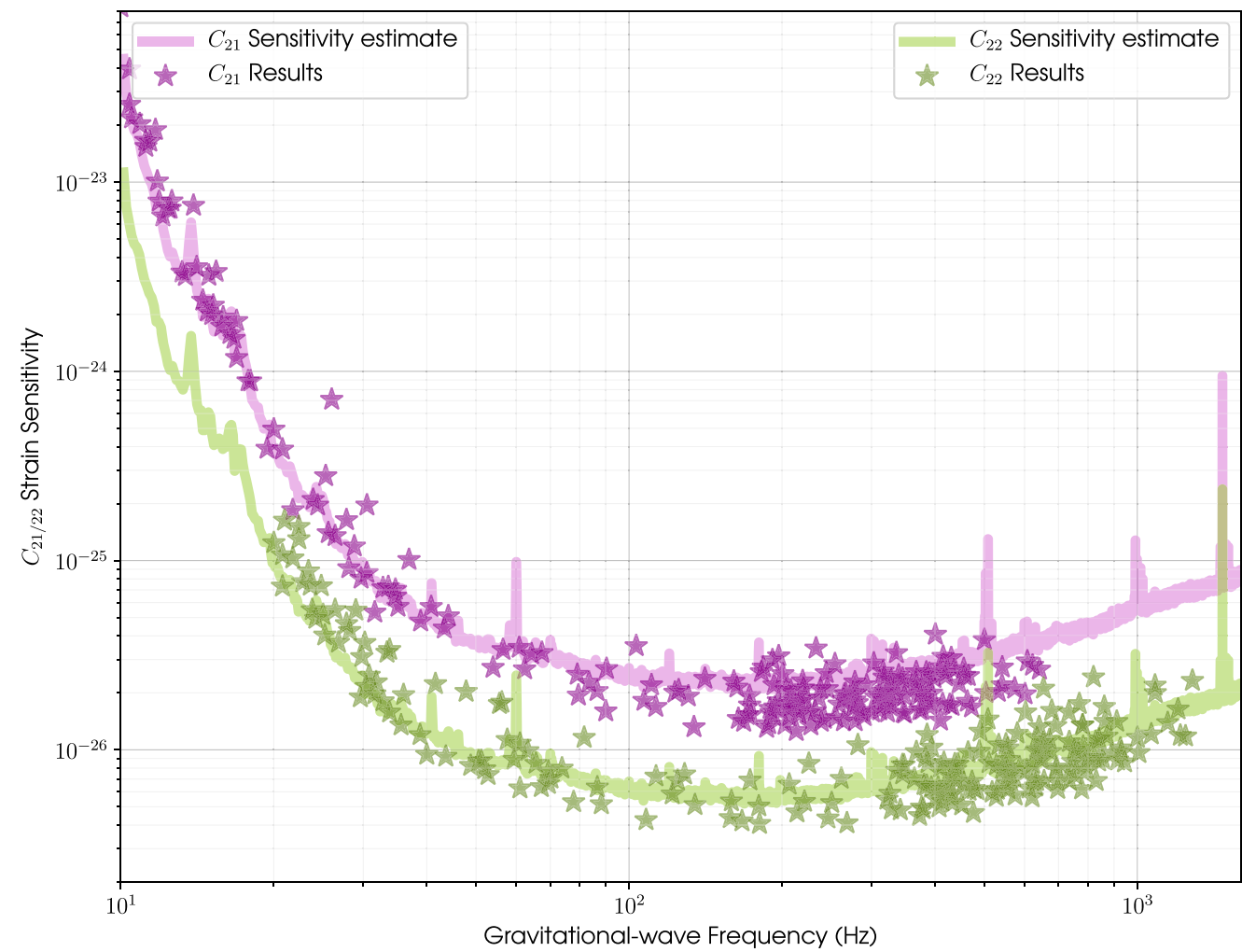

Figure 1. Upper limits on $C_{21}$ and $C_{22}$ for 221 pulsars. The stars show the observed $95 \%$ credible upper limits on observed amplitudes for each pulsar. The solid lines show an estimate of the expected sensitivity of the searches.

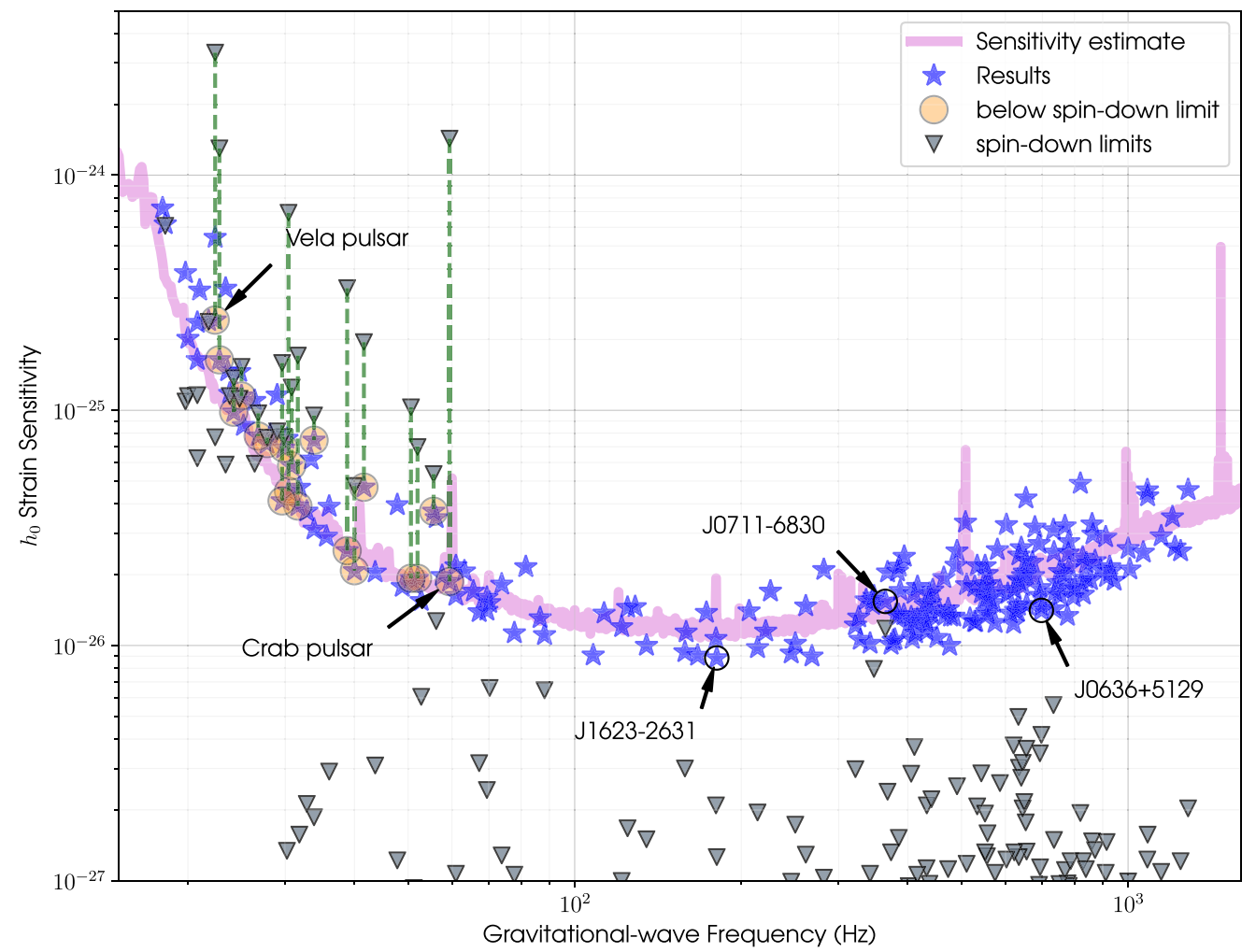

Figure 2. Upper limits on $h_{0}$ for 221 pulsars. The stars show the observed $95 \%$ credible upper limits on observed amplitude for each pulsar. The solid line shows an estimate of the expected sensitivity of the search. Triangles show the limits on gravitational-wave amplitude derived from each pulsar's observed spin-down. 


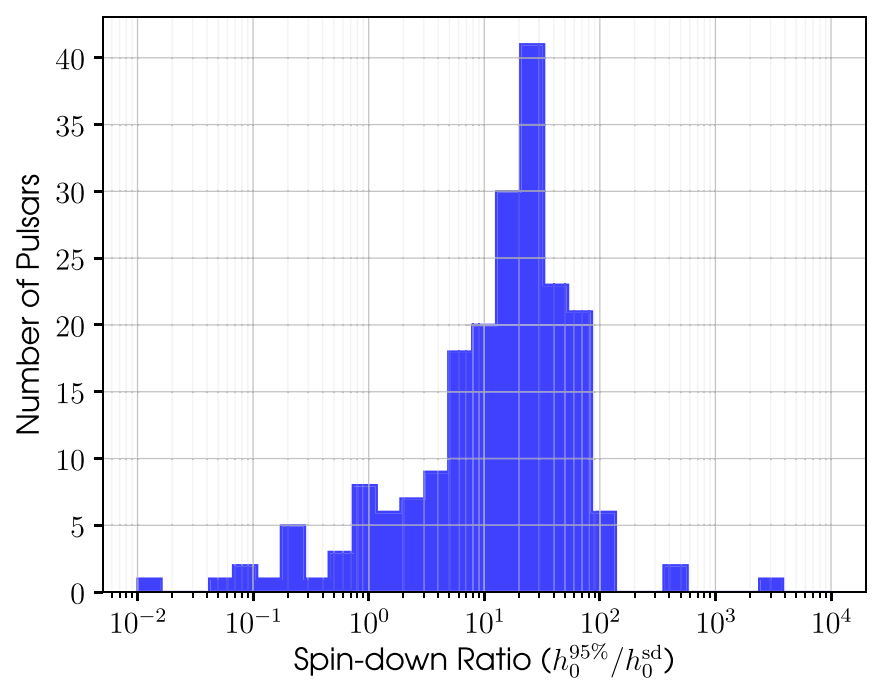

Figure 3. Histogram of ratios of upper limits on $h_{0}$ compared to the spin-down limit.

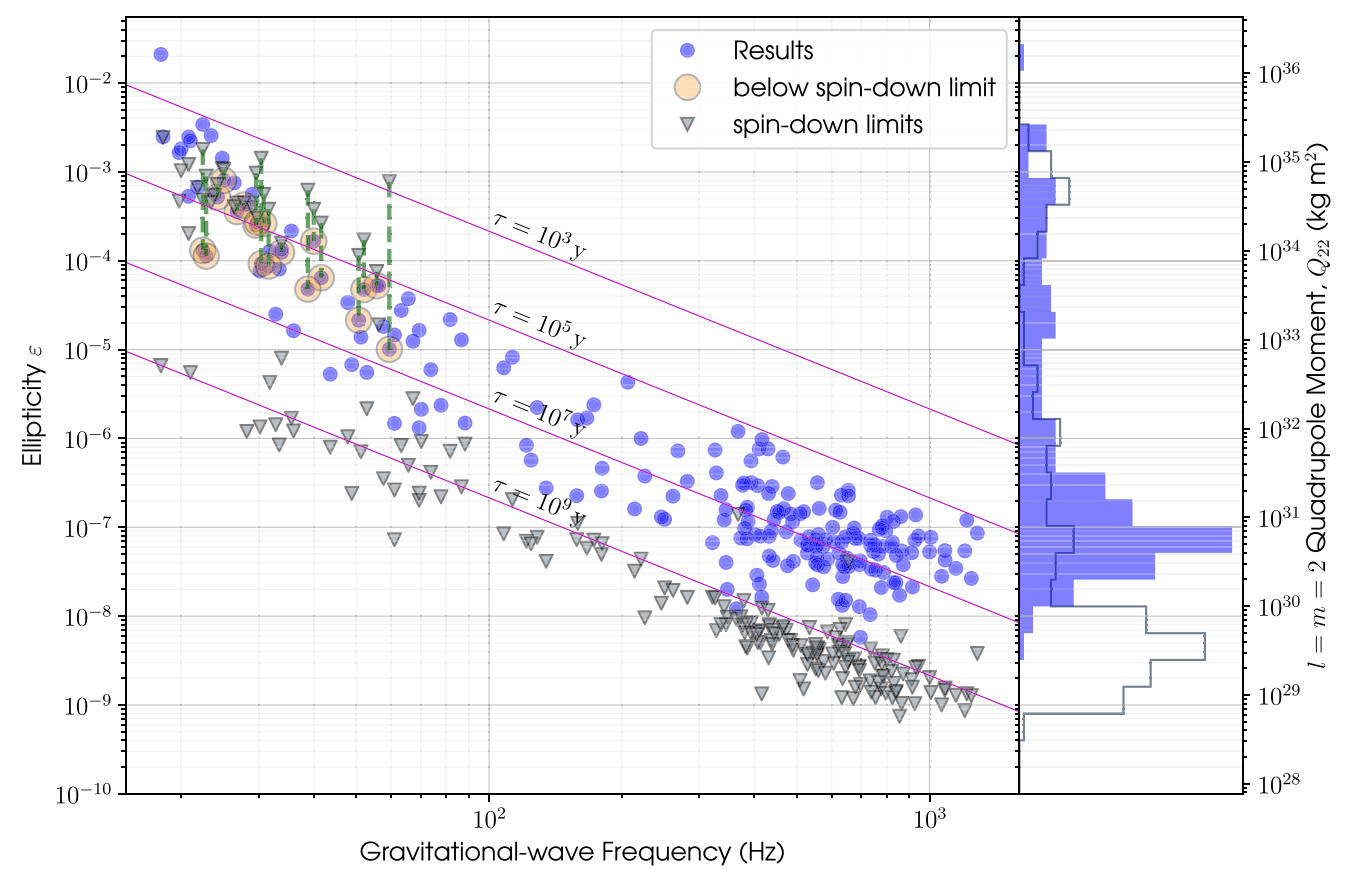

Figure 4. Upper limits on mass quadrupole $Q_{22}$ and fiducial ellipticity $\varepsilon$ for 221 pulsars. The filled circles show the limits as derived from the observed upper limits on the gravitational-wave amplitude $h_{0}$ assuming the canonical moment of inertia and distances. Triangles show the limits derived from each pulsar's observed spindown. The diagonal lines show contours of equal characteristic age $\tau$ assuming that braking is entirely through gravitational-wave emission. The distributions of these limits are also show in histogram form to the right of the figure, with the filled and open histograms showing our observed limits and the spin-down limits, respectively.

\section{Reference}

Abbott, B. P., Abbot, R., Abbott, T. D., et al. 2019, ApJ, 879, 10 